\title{
La presencia de síndrome metabólico se asocia a un aumento del riesgo cardiovascular en pacientes con diabetes tipo 2
}

\section{Objetivo}

Analizar la presencia de síndrome metabólico como variable independiente en relación al riesgo de eventos cardiovasculares en una población de paciente con diabetes tipo 2 .

Diseño

Estudio de cohortes prospectivo.

Lugar

Alcañiz, Prov de Teruel, Comunidad autónoma de Aragón, España

\section{Pacientes y métodos}

Se reclutaron pacientes con diabetes tipo 2 que se atendían en el servicio de endocrinología del hospital local. La población de referencia era de 70.000 personas. Se excluyeron a los pacientes con proteinuria, y ausencia de datos. De los 463 candidatos posibles participaron en el estudio 318. La duración media del seguimiento fue 4,6 años.

\section{Medición de los factores pronósticos}

Se constató la presencia inicial de componentes del síndrome metabólico (hipertensión, colesterol HDL y trigicéridos, índice de masa corporal, relación cintura cadera y microalbuminuria) y de diabetes 2 según la definición de la Organización Mundial de la Salud. Además se registró el sexo, edad, hábito tabáquico y el tipo de tratamiento que recibían los pacientes en relación a su diabetes.

\section{Medición de resultados}

El resultado primario fue una combinación de eventos coronarios (nueva angina o infarto agudo de miocardio: IAM), cerebrovasculares (accidente isquémico transitorio o accidente cerebrovascular) y amputación de extremidades inferiores. Los resultados secundarios fueron los eventos coronarios o cerebrovasculares reportados en forma separada. Se evaluó la asociación entre síndrome metabólico o alguno de sus componentes y la aparición de nuevos eventos.

\section{Resultados}

La prevalencia inicial de síndrome metabólico fue $77 \%$. Las tasas de enfermedad cardiovascular global, coronaria y cerebrovascular, expresadas por cada 1.000 pacientes/año, fueron: $14,0,5,6$ y 8,4 en pacientes sin síndrome metabólico y 33,3, 20,7 y 11,7, respectivamente en pacientes con síndrome metabólico $(p=0,058$ para eventos totales y $p=0,05$ para eventos coronarios). En el análisis multivariable la presencia simultánea de los cuatro componentes del síndrome metabólico incrementó significativamente el riesgo de enfermedad global $(R R=5 ;$ IC $95 \% 1,6-15,9)$ y coronaria $(R R=$ 7,4 ; IC 95\% 1,3-41,1) y no así la enfermedad cerebrovascular. Este análisis fue ajustado por edad, tabaquismo y niveles de hemoglobina glicosilada.

Cuadro 1:

\begin{tabular}{l|c|c|c}
\hline Síndrome metabólico & \multicolumn{3}{|c}{ Número de eventos cada 1000 pacientes/año } \\
& $\begin{array}{c}\text { Enfermedad } \\
\text { cardiovascular global }\end{array}$ & $\begin{array}{c}\text { Enfermedad } \\
\text { coronaria }\end{array}$ & $\begin{array}{c}\text { Enfermedad } \\
\text { cerebrovascular }\end{array}$ \\
\hline Ausente & 14 & 5,6 & 8,4 \\
\hline Presente & 33,3 & 20,7 & 11,7 \\
\hline
\end{tabular}

\section{Conclusiones}

Independientemente de la edad, el tabaquismo o el mal control de la enfermedad, la presencia de los cuatro componentes del síndrome metabólico se asoció a mayor riesgo de enfermedad cardiovascular en pacientes con diabetes tipo 2 .

\section{Comentario}

Según Reaven, el "inventor" de la resistencia insulínica "el valor del concepto de resistencia insulínica radica en que provee un marco conceptual para colocar una serie de eventos aparentemente no relacionados biológicamente en una construcción fisiopatológica. En contraste, el síndrome metabólico (SM) ha sido introducido como una categoría diagnóstica para identificar sujetos, que al cumplir una serie de parámetros poseen mayor riesgo cardiovascular. Estos individuos se benefician al modificar sus estilos de vida. Consecuentemente, el concepto de SM permite un abordaje pragmático para obtener un mejor resultado clínico'.

Resumiendo, el SM es una entidad clínica conformada por varios componentes (hipertensión, dislipemia, obesidad, alteraciones en el metabolismo de la glucosa y microalbuminuria) pudiendo estos parámetros explicarse por la presencia basal de resistencia a la insulina (RI). Numerosos cuadros pueden provocar RI: síndrome de ovario poliquístico, exceso de cortisol y, más frecuentemente, obesidad. Es decir, tener obesidad es el factor desencadenante de la RI y la RI es a su vez disparadora de múltiples fenómenos que determinan en algunos pacientes la aparición de síndrome metabólico.

La diabetes tipo 2 es el punto final de dos vías que habitualmente se dan simultáneamente, la primera es la disminución de la secreción insulínica por "agotamiento pancreático" determinada fundamentalmente por factores genéticos. La segunda, es la resistencia a la insulina. Esta a su vez se encuentra determinada por múltiples factores, siendo los más importantes el sedentarismo y la obesidad.

Ahora, ¿es el SM una categoría de riesgo útil en los pacientes diabéticos? En este sentido, estos dos trabajos han intentado evaluar si los pacientes con diabetes tipo 2 tienen mayor riesgo de enfermar o morir por enfermedad cardiovascular según cumplan o nó con los parámetros de síndrome metabólico (SM).

Primero, un breve comentario acerca del estudio español, que incluyó un pequeño número de pacientes y evaluó incidencia de enfermedad cardiovascular (ECV). Si bien el estudio mostró que los pacientes con diabetes tipo 2 y SM tienen más riesgo de ECV quienes no lo tienen, es cuestionable su validez interna, básicamente la no especificación de la condición de "libre de enfermedad" cardiovascular (ECV) al inicio del estudio. Otro punto cuestionable del estudio de Gimeno Orna y col. son los parámetros utilizados para definir el SM. Los valores de tensión arterial $(160 / 90 \mathrm{mmHg})$ y microalbuminuria (30mg en $24 \mathrm{~h}$ ) fueron más elevados en el estudio

Alejandrina Lo Sasso [ Unidad de Medicina Familiar y Preventiva. Hospital Italiano de Buenos Aires. ] 


\section{La presencia de de síndrome metabólico en pacientes diabéticos tipo 2 no se asoció a una mayor tasa de mortalidad cardiovascular}

\section{Objetivo}

Definir la asociación entre enfermedad cardiovascular y la presencia del síndrome metabólico en una población de diabéticos tipo 2 .

\section{Diseño}

Estudio de cohortes prospectivo.

\section{Lugar}

Pueblo de Casale Monferrato, Italia.

\section{Pacientes}

Se siguieron 1565 pacientes diabéticos tipo 2 entre los años 1988 y 2001, con una mediana de seguimiento de 8,21 años.

\section{Evaluación de factores pronósticos}

Se relevó información acerca del tiempo de diagnóstico de diabetes, proveedor del tratamiento (especialista, médico general) y tipo de tratamiento. Se cuantificaron todas las variables del síndrome metabólico (peso, diámetro abdominal, tensión arterial, perfil lipídico, microalbuminuria) así como la hemoglobina glicosilada. Además, se evaluó la presencia de anormalidades electrocardiográficas compatibles con enfermedad coronaria al inicio del estudio.

\section{Medición de resultados principales}

La variable principal fue la comparación del riesgo relativo de muerte (global y cardiovascular) según la presencia del síndrome metabólico o alguno de sus componentes.

\section{Resultados}

La prevalencia basal de síndrome metabólico fue 75,6\%. Hubo 685 muertes, 520 en el grupo con síndrome metabólico (256 de causa cardiovascular). En el grupo sin síndrome metabólico hubo 165 muertes, 85 de origen cardiovascular.
El riesgo relativo (expresados como hazard ratio*) de muerte global o por enfermedad cardiovascular fue similar en ambos grupos de pacientes (con o sin síndrome metabólico) aún ajustando por edad, tabaquismo, sexo, niveles de colesterol o presencia de enfermedad coronaria $(\mathrm{RR}=1,06 \quad \mathrm{IC} \%$ \% $0,86-1,3)$. En aquellos pacientes que presentaban diabetes y sólo un componente más del síndrome metabólico, el riesgo relativo de muerte cardiovascular fue 2,8 (IC95\% 1,11-7,11). Ver cuadro 2.

\section{Cuadro 2:}

\begin{tabular}{|c|c|c|c|c|c|}
\hline & \multicolumn{2}{|c|}{ Mortalidad global } & \multicolumn{2}{|c|}{ Mortalidad cardiovascular } \\
\hline & & $\begin{array}{l}\mathrm{N}^{0} \text { de muertes } \\
\text { (1000/ } \\
\text { personas-año) }\end{array}$ & $\begin{array}{l}\text { Hazard ratio } \\
\text { (IC95\% )* }\end{array}$ & $\begin{array}{l}\mathrm{W}^{0} \text { de muertes } \\
\text { (1000/ } \\
\text { personas-año) }\end{array}$ & $\begin{array}{l}\text { Hazard ratio } \\
\text { (IC95\% )* }\end{array}$ \\
\hline \multirow{2}{*}{$\begin{array}{l}\text { Síndrome } \\
\text { metabólico }\end{array}$} & Ausente & $165(64,7)$ & 1 & $85(33,4)$ & 1 \\
\hline & Presente & $520(63,0)$ & $1,06(0,86-1,30)$ & $256(31,0)$ & $0,95(0,71-1,28)$ \\
\hline \multirow{4}{*}{$\begin{array}{l}\text { Múmero de } \\
\text { componentes }\end{array}$} & o & $30(58,7)$ & 1 & $11(21,5)$ & 1 \\
\hline & 1 & $135(66,2)$ & $1,37(0,83-2,25)$ & $74(36,3)$ & $2,8(1,11-7,11)$ \\
\hline & 2 & $249(68,3)$ & $1,36(0,85-2,21)$ & $116(31,8)$ & $2,21(0,89-5,49)$ \\
\hline & $>2$ & $271(58,8)$ & $1,25(0,78-2,02)$ & $0140(30,4)$ & $2,46(0,99-6,07)$ \\
\hline
\end{tabular}

* ajustado por edad, sexo, tabaquismo, colesterol, presencia previa de ECV y $\mathrm{Hb}$ glicosilada.

\section{Conclusiones}

Los autores concluyen que la incidencia de síndrome metabólico es alta en la población de diabéticos tipo 2 . Sin embargo, la presencia del síndrome no aumentaría la mortalidad cardiovascular ni la global. Un dato observacional no explicado por los autores es el aumento del riesgo cardiovascular en los pacientes diabéticos tipo 2 con un sólo componente del síndrome metabólico.

Fuente de financiamiento: Ministerio della Istruzione Università e Ricerca Scientifica e Tecnologica (MIUR) Italia. Asociación Italiana para la investigación en cáncer y empresa San Paolo/FIRMS español. ¿Cuál es la importancia de este dato? Simplemente que los pacientes que cumplían estos parámetros, es decir los catalogados como portadores de SM, pueden haber estado más enfermos que los del estudio italiano y eso determinar el resultado positivo que ellos obtuvieron.

En contraste con el español, el estudio italiano incluyó una enorme cantidad de pacientes y obtuvo resultados negativos respecto de diferencias en la mortalidad. Sin embargo, debemos ser cautelosos en las conclusiones ya que si bien compartimos cierta parte del origen étnico con el pueblo italiano, sus conclusiones pueden ser difíciles de extrapolar a nuestro medio.

A pesar de estas diferencias y tomando ambos estudios como válidos, ¿cómo explicar que tengan resultados de valor opuesto? La primera salvedad a esta afirmación es que los estudios midieron cosas diferentes. El español evaluó incidencia de ECV, mientras que el estudio italiano cuantificó mortalidad. El estudio italiano no informa si a pesar de tener igual mortalidad en ese período, la prevalencia de enfermedad era diferente entre ambos grupos. Aún así, si consideramos que a mayor incidencia corresponde mayor mortalidad y que a igual mortalidad corresponde una incidencia de enfermedad similar, los estudios tendrían resultados diferentes.
Numerosos estudios muestran que tener resistencia a la insulina o SM confiere mayor riesgo de diabetes y mayor riesgo cardiovascu$\operatorname{lar}^{2,3}$. Mientras el paciente no es diabético tiene claro sentido valorar la presencia de SM, no tanto como rótulo sino como una valoración global de su riesgo futuro, que será estimado como mayor. Este punto no queda tan claro en la población de diabéticos.

\section{Conclusiones del comentador}

Estudios de resultados opuestos sugieren que hacen falta más investigaciones. De todas formas, las metas de tensión arterial y colesterol recomendadas para pacientes diabéticos no se modifican en presencia de SM, ya que el riesgo de todo paciente diabético de enfermar o morir por ECV se asume como los suficientemente alto como para avalar metas estrictas. Sin embargo, en aquellos pacientes que presentan SM pero aún no son diabéticos existe la posibilidad de fomentar hábitos de vida saludable (dieta, actividad física) para disminuir la probabilidad de desarrollo de la enfermedad.

\section{Alejandrina Lo Sasso [ Unidad de Medicina Familiar y Preventiva. Hospital Italiano de Buenos Aires. ]}

Lo Sasso A. La presencia de Síndrome Metabólico se asoció a un aumento del riesgo cardiovascular en pacientes con diabetes tipo 2. La presencia de síndrome metabólico en pacientes diabéticos tipo 2 no se asoció a una mayor tasa de mortalidad cardiovascular. Evid actual práct. ambul 2005:8:110-111. Comentado de: Gimeno Orna JA, Lou Arnal LM, MolineroHerguedas E y col. Metabolic syndrome as a cardiovascular risk factor in patients with type 2 diabetes. Rev Esp Cardiol. 2004 Jun;57(6):507-13.PMID: 15225497. Bruno G, Merletti F, Biggeri A y col. Metabolic Sindrome as a Predictor of All-Cause and Cardiovascular Mortality in type 2 Diabetes. The Casale Monferrato Study. Diabetes Care. 2004 Nov;27(11):2689-94. PMID: 15505006.

\section{Bibliografía:}

1. The Metabolic Syndrome: Requiescat in Pace. Gerald M. Reaven, Clinical Chemistry, March, 2005, 51: 931-938.

2. The metabolic syndrome as predictor of type 2 diabetes: The San Antonio Heart. C. Lorenzo, M. Okoloise, K. Williams y col., Diabetes Care; Nov $2003 ; 26,11$. 3. The Metabolic Syndrome and Total and Cardiovascular Disease Mortality in Middle-aged Men. Lakka HM, Laaksonen DE, Lakka TA. JAMA 2002, 288:2709 2716 . 